\title{
QUALITY OF SERVICE AWARE OF CROSS LAYER APPROACH ON ADAPTIVE GATEWAY DISCOVERY SCHEME FOR MOBILE AD HOC NETWORKS
}

\author{
${ }^{1}$ Palani, K. and ${ }^{2}$ P. Ramamoorthy \\ ${ }^{1}$ Department of Computer Science and Engineering, \\ Tamilnadu College Engineering, Coimbatore, Tamilnadu, India \\ ${ }^{2}$ Department of Electronic Communication and Engineering, \\ SNS College of Technology, Coimbatore, Tamilnadu, India
}

Received 2013-06-22, Revised 2013-08-22; Accepted 2013-08-28

\begin{abstract}
The Quality of Service (QoS) providing in Mobile Ad hoc networks has been obtaining increasing importance, specifically Consider the domains of application for networks in disaster environments and defense ventures, QoS is performing level of a service recommended by the network to the consumer. Mobile Ad hoc Networks (MANET) are necessary because of wireless infrastructure network is complicated to setup for providing communication over huge areas, with the initiation of the Internet, requirement for global communication is also becoming an essential requirement for MANETs, Hence; Internet connectivity in MANETs has received considerable attention in the recent years. Routing packets effectively inside and outside a MANET is a challenge and much endeavor has been given to the design of routing protocols to attain higher Packet Delivery Ratio (PDR) over Intra-MANET and Internet-MANET. Though, most of these routing protocols use information obtainable at the network layer to route packets. This study proposed to Contention Aware QoS based Adaptive routing Protocol by manipulating the information gained at the link layer as well as to the information obtainable at the network layer to achieve higher PDR compared to existing protocols, in simulations shown that QoS aware cross layer approach can achieve higher PDR for Internet-MANET traffic.
\end{abstract}

Keywords: Contention Aware of QoS Based Adaptive Routing Protocol (CAQAP), Intra-MANET, Internet-MANET, Packed Delivery Ratio and Gateway Discovery

\section{INTRODUCTION}

Wireless ad hoc is an independent network with moveable nodes, which can transmit with each other on several hops and worked without any fixed infrastructure. In hybrid MANET, integration provides an efficient data transmission. All the nodes are alike in the mobile ad-hoc networks; there is no controlling entity like an access point in the wired networks such as the Internet. It is formed spontaneously in the areas where creating an infrastructure network causes delay until the work as well as increases the cost of utilization. It is united of portable mobile nodes like as notebooks, palmtops, PDAs and handheld mobile phones. A main issue in mobile ad-hoc networks is the adaptable and the easy deployment and the selfconfigurable of the mobile ad-hoc networks. The above attributes are most important in a mobile ad-hoc network. A node of hybrid MANET contains connectivity to the infrastructure networks for receiving data and multimedia services. Routing among the MANET and the Internet was first proposed by Perkins (1996), where MANET devices gain access to the Internet via special MANET devices running both

Corresponding Author: Palani, K., Department of Computer Science and Engineering, Tamilnadu College Engineering, Coimbatore, Tamilnadu, India 
Mobile IP and ad hoc routing protocol, performing as gateways between the MANET and the Internet.

A many researchers work has been done on hybridMANET architecture, gateway discovery mechanisms for discussed on Jonsson et al. (2000) and Ergen and Puri (2002), address configuration Sun et al. (2002) and Nilsson et al. (2002) reducing routing overhead for gateway discovery and achieving high PDR focus on Hwang et al. (2005). PDR is a very important measure in hybrid MANETs because higher PDR means reduced congestion and disruption during communication between MANET nodes. Even though some researchers have been carried out to achieve high PDR, no studies has so far focused on showing PDR over InternetMANET traffic separately from Intra-MANET traffic. The key problem of this is that when packet loss occurs, it is impossible to tell whether lower PDR was due to loss in Intra-MANET traffic, Internet- MANET traffic or together traffic. Packet loss of Internet-MANET and Intra-MANET traffic occur at different rates. This is because all Internet-MANET packets travel through the gateway, thus heavily overloading the gateway with traffic and causing excessive packet loss. Therefore, packets loss of Internet-MANET is always higher than packet loss of Intra-MANET traffic. An objective here is to put forward QoS based protocol such that high PDR can be obtained over Internet-MANET traffic. In this work, show the performance of PDR of Intra-MANET traffic and Internet-MANET traffic and also state what percentage each of these traffic signify during simulation.

The rest of paper is structured as follows. Section II cites related work in the area of Internet connectivity in MANETs. In Section III, describe the proposed for gateway discovery scheme and IV describes the metric based cross-layer approach. Section V shows result analysis of Contention Aware QoS based Adaptive Protocol through NS2 simulation. Section VI presents concluding remark with future works.

\section{RELATED WORK}

Many research efforts various architectures have been proposed to connect a MANET to the Internet via a gateway, Ding (2008), but focus mainly on ip based wireless system architectures that employ Mobile IP because it is the next step to be implemented to achieve ubiquitous communication Akyildiz et al. (2004). Proposed the Mobile IP integrated architectures and its main challenge was to determine whether packets need to be routed to the Internet via a gateway and if so, how to reach the gateway. Different architectures were developed for connecting MANETs to the Internet, Tseng et al. (2003); Ammari and El-Rewini (2004); Ergen and Puri (2002) and Ratanchandani and Kravets (2003). Jonsson et al. (2002) developed MIPMANET, one of the very first detailed works on MANET Internet integrated architecture using Mobile IP. Tseng et al. (2003) and Ergen and Puri (2002) also developed similar architectures, however, they do not have proper IPv6 specifications developed.

Ammari and El-Rewini (2004), multiple gateway architecture was proposed for MANET nodes to communicate with the Internet but the architecture suffers from redundant signaling overhead because of multiple gateways Ding (2008). A considerable amount of work had been done on gateway discovery. Studies on gateway discovery and address configuration were discussed in Sun et al. (2002); Xi and Bettstetter (2002); Nilsson et al. (2002) and Wakikawa et al. (2006). Other works included gateway discovery such that routing overhead can be reduced and higher packet delivery can be obtained Lee et al. (2003); Ruiz and Gomez-Skarmeta (2005); Hoang et al. (2004) and Hwang et al. (2005). Most of the above works described connecting a MANET to the Internet using Mobile IP. A major limitation of this architecture is that mobile nodes have to go through unnecessary gateway registrations Ding (2008), when they are not experiencing interMANET mobility but simply seeking Internet connectivity. Only a few works Xi and Bettstetter (2002); Nilsson et al. (2002); Wakikawa et al. (2006) and Hwang et al. (2005) explicate IPv6 MANETs where nodes can obtain Internet connectivity without the aid of Mobile IP but still have provisions for incorporating Mobile IP. This architectures proposed on $\mathrm{Xi}$ and Bettstetter (2002); Nilsson et al. (2002); Wakikawa et al. (2006) and Hwang et al. (2005) can perform better when quick Internet connectivity is required, because unnecessary Mobile IP gateway registrations are not needed. However, a determining factor of such quickness is the ad hoc routing protocol used in the MANET.

Two major classes of routing protocols, reactive and proactive, are typically used in MANETs to determine where and how packets need to be routed. In reactive protocols, the time taken to determine whether packets need to be routed to the Internet via a gateway and the route acquisition time to a gateway is very large because routes are not readily available. Delay caused by such route acquisition time causes PDR to fall in InternetMANET traffic, thus reactive protocols are not suitable for achieving high PDR when routing packets to and from the Internet. In Proactive protocols, also known as 
table-driven protocols, packets designed within a MANET are immediately routed because all MANET destinations are readily available. Packets designed outside the MANET are also readily routed towards the gateway, Wakikawa et al. (2006), also known as default route and forwarded accordingly. Thus, proactive protocols are more suitable for quick Internet connectivity. To ensure high PDR, based on this study at existing proactive routing protocol based architecture. Hwang et al. (2005) proposed an IPv6 based self-addressing routing protocol where MANET nodes form a tree overlay rooted at the gateway. Here packets designed for the Internet are routed towards the gateway using hierarchical routing and packets designed within the MANET are routed using soft state routing Hwang et al. (2005).

Most real world implementations comprise of lowmobility small-scale MANETs Kiess and Mauve (2007). Hamadan et al. (2003) proposed a solution, which provides Internet connectivity to ad hoc networks by modifying the AODV routing protocol. Three methods of gateway discovery for a mobile node to access the Internet are provided: proactive, reactive and hybrid approach. All of them are based only on the number of physical hops to gateway as the metric for the gateway selection. Bin et al. (2005) proposed an adaptive gateway discovery scheme that can dynamically adjust the TTL value of Agent Advertisements (GWADV messages) according to the mobile nodes MANET Internet traffic and their related position from Internet Gateways with which they registered. This protocol provides Internet access to MANET mobile nodes using mobile IP. In all the protocols discussed above, to obtain Internet connectivity, the main challenge is to locate a path from a node to the gateway and preferably a path with minimum hops to the gateway as it usually exhibits less routing delay and also may lead to higher PDR over a period of time. So that this is not a sufficient measurement to achieving high PDR while receiving or forwarding packets to and from the gateway because minimum hops to the gateway doesn't take into consideration the drop in PDR due to packet loss induced by network congestion or contention along that path. It is not possible to determine such congestion or contention from the network layer, as a result, typical ad hoc routing protocols suffers the consequences of packet loss. The core contribution of this work is to develop a congestion-aware MANET routing protocol that will provide global connectivity and improved Quality of Service (QoS) for the next-generation applications and devices Akyildiz et al. (2004).

In order to determine congestion or contention, crosslayer routing has recently been proposed. In cross-layer routing, different layers of the Open System Interconnection (OSI) model share information gathered at each layer to improve performance in terms of packets delivery, routing delay, routing overhead and various other performance measures. In the past few years, a number of studies have been done on cross-layer routing in MANETs to achieve high packet delivery ratio. Denko et al. (2007) described a cross-layer routing protocol where specially selected mobility-aware nodes called brokers, publishers and subscribers route packets based on node mobility determined through link failures detected at the link layer. Song and Fang (2006) modified Dynamic Source Routing (DSR) by allowing nodes to determine stable paths from link layer frame collisions and channel usage determined from Network Allocation Vector (NAV). However, all these cross-layer techniques use reactive protocol, which as discussed earlier, are not suitable for achieving high PDR over internet-MANET communication. Karbaschi and Fladenmuller (2005) proposed a cross-layer approach on a proactive protocol to measure the quality of a path between any two given nodes in a MANET based on the amount of contention arising at each link along that path. It was shown that higher PDR could be obtained if packets were traveling along a path having no or less contention compared to a contention prone path. In addition to the contention measure, congestion along any path also adversely affects the PDR. In this study show that CAQAP can outperform both Hybrid and Adaptive in terms of PDR in small-scale low-mobility networks.

\section{PROPOSED FOR GATEWAY DISCOVERY SCHEME}

In this section provide an analytical model to compute the gateway discovery overhead which is caused by the reactive, proactive, hybrid, adaptive schemes. Let's assume that there are $\mathrm{N}$ nodes in a square lattice covering a certain area, as in Fig. 1. Each vertex of the lattice represents one and only one node. Some of them, NGW, are gateways placed in the corners of the lattice. Then, the model have $\mathrm{N}_{\mathrm{adhoc}}=\mathrm{N}-\mathrm{N}_{\mathrm{GW}}$ ad hoc nodes. There are $S$ traffic sources which are uniformly distributed in the network, so that every node has the same probability to be a source. Given that an interested in modeling gateway discovery, assume that receivers are in the Internet. During the time interval $t$ under consideration, all sources send constant bit rate traffic to the fixed nodes through the gateways. The metric used to choose a route to the gateway is the hop count, since it is common to all solutions and allows for a fair comparison. Therefore, every node selects the nearest gateway to communicate with hosts in the Internet. Under these circumstances, assume that there are $\mathrm{N}_{\text {adhoc }}=$ $\mathrm{N}-\mathrm{N}_{\mathrm{GW}}$ potential nodes which can use a given gateway in their default routes. 


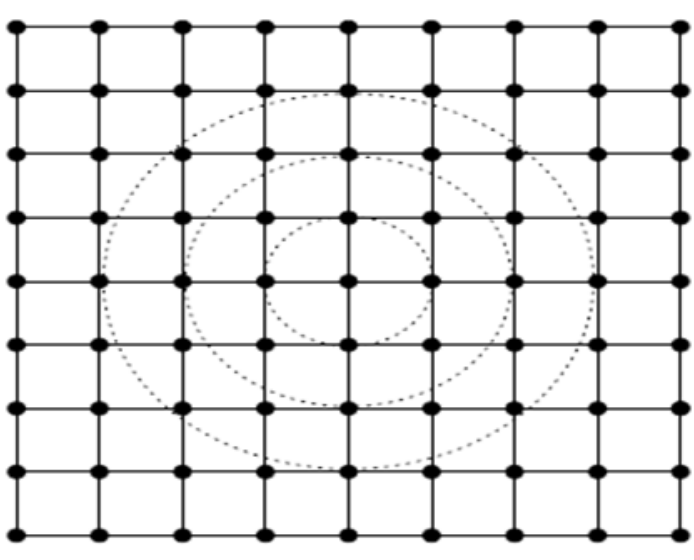

Fig. 1. Square lattice used in the proposed analytical model

Whenever a source wants to reactively discover a gateway, it floods the network with a RREQ I message after that, every gateway sends a RREP I reply unicasted to the source. Since the gateways are in the corners of the lattice, it is easy to check that the mean path length is $\sqrt{\mathrm{N}}-1$. Then, the overhead of the reactive gateway discovery for every source is given by Equation 1:

$$
\Omega_{\mathrm{r}-\mathrm{gw}}=\mathrm{N}_{\mathrm{adhoc}}+\mathrm{N}_{\mathrm{GW}}(\sqrt{\mathrm{N}}-1)
$$

Link breaks are mainly due to the effects of mobility. When a link between two nodes of an active route breaks, the node that detects it notifies the source by sending a RERR message. This overhead is similar for every approach and much lower than the gateway discovery function overhead. Moreover, that message is part of the routing protocol rather than the interconnection mechanism itself. Therefore do not take it into account. The number of link breaks in a given scenario and number of route discoveries which are caused by those breaks, can be better determined through a simulated analysis. Figure 2 shows the mean number of route discoveries per second which are issued for a range of scenarios with different number of sources and gateways. To get this result, 10 different runs for each case have been performed during $500 \mathrm{~ms}$ and obtain see how the number of route discoveries (rd $\left(\mathrm{S}, \mathrm{N}_{\mathrm{GW}}\right)$ ) decreases for the cases of 5 and 6 gateways, which is due to the shorter mean path length in those scenarios.

Finally, Equation 2 gives the overhead of the reactive scheme as the overhead of discovering the gateway reactively multiplied by the number of such route discoveries that need to be done during the time interval t:

$$
\Omega_{\mathrm{r}}=\Omega_{\mathrm{r}-\mathrm{gw}} \cdot \mathrm{t} \cdot\left(\mathrm{rd}\left(\mathrm{S}, \mathrm{N}_{\mathrm{GW}}\right)\right)
$$

Let us continue an analysis with the overhead of the proactive scheme, where GWADV messages are sent by the gateways to the whole ad hoc network. For each gateway, the associated overhead is of $\mathrm{N}_{\mathrm{adhoc}}+1$ messages; one forwarding by each of the $\mathrm{N}_{\text {adhoc }}$ nodes plus the first message which is sent by the gateway itself. Let $\lambda_{\text {adv }}$ be the rate at which GWADV messages are sent out. The overhead of the proactive solution can be obtained as in Equation 3:

$\Omega_{\mathrm{p}}=\lambda_{\mathrm{adv}} \cdot \mathrm{t} \cdot\left(\mathrm{N}_{\mathrm{ad} \mathrm{hoc}}+1\right) \cdot \mathrm{N}_{\mathrm{GW}}$

The hybrid gateway discovery scheme has an overhead which is a combination of the reactive and proactive protocols. As we showed, the mean path length is $\sqrt{\mathrm{N}}-1$. Thus, it makes no sense sending GWADV messages at more than $\sqrt{\mathrm{N}}-1$ hops because other gateways will be covering the area beyond that TTL (assuming gateways are in the corners). The number of nodes which are at an scope of s hops from any gateway is approximated by Equation 4, with $s \in[0, \sqrt{\mathrm{N}}-1]$ :

$$
\mathrm{N}_{\mathrm{r}}^{\mathrm{GW}}(\mathrm{s}) \simeq \sum_{\mathrm{j}=1}^{\mathrm{s}}(\mathrm{j}+1)=\frac{\mathrm{s}(\mathrm{s}+3)}{2}
$$

For a given scope s configured at each gateway, the probability for a node to receive a GWADV message from any of the gateways can be computed as shown in Equation 5. It is an approximated expression, since not all the gateways necessarily cover the same number of ad hoc nodes:

$$
\mathrm{P}_{\mathrm{c}(\mathrm{s})} \simeq \frac{\mathrm{N}_{\mathrm{r}}^{\mathrm{GW}}(\mathrm{s}) \cdot \mathrm{N}_{\mathrm{GW}}}{\mathrm{N}_{\text {ad hoc }}}
$$

If we denote $\mathrm{N}_{\mathrm{c}}$ as the number of sources being covered by any gateway when using a scope of $\mathrm{s}$ hops, then $\mathrm{N}_{\mathrm{c}}$ is a random variable obeying a binomial distribution $\mathrm{B} \sim(\mathrm{S}, \mathrm{Pc}(\mathrm{s}))$. Thus, the mean number of sources being covered when gateways use a scope of $\mathrm{s}$ hops can be computed as $\left.\mathrm{E}\left[\mathrm{N}_{\mathrm{c}}\right]=\mathrm{S} . \mathrm{P}_{\mathrm{c}(\mathrm{s})}\right)$. So, the overall overhead of the hybrid approach consists of the proactive sending of GWADV messages up to $s$ hops, plus the reactive discovery of a gateway by those sources not covered by the GWADV messages (Equation 6):

$$
\begin{aligned}
& \Omega_{\mathrm{h}}^{\mathrm{s}}=\lambda_{\mathrm{adv}} \cdot \mathrm{t} \cdot\left(\mathrm{N}_{\mathrm{r}}^{\mathrm{GW}}(\mathrm{s})+1\right) \cdot \mathrm{N}_{\mathrm{GW}}+ \\
& \Omega_{\mathrm{r}-\mathrm{gw}} \cdot \mathrm{t} \cdot\left(\mathrm{rd}\left(\mathrm{S}, \mathrm{N}_{\mathrm{GW}}\right)\right) \cdot\left(1-\mathrm{P}_{\mathrm{c}(\mathrm{s})}\right)
\end{aligned}
$$




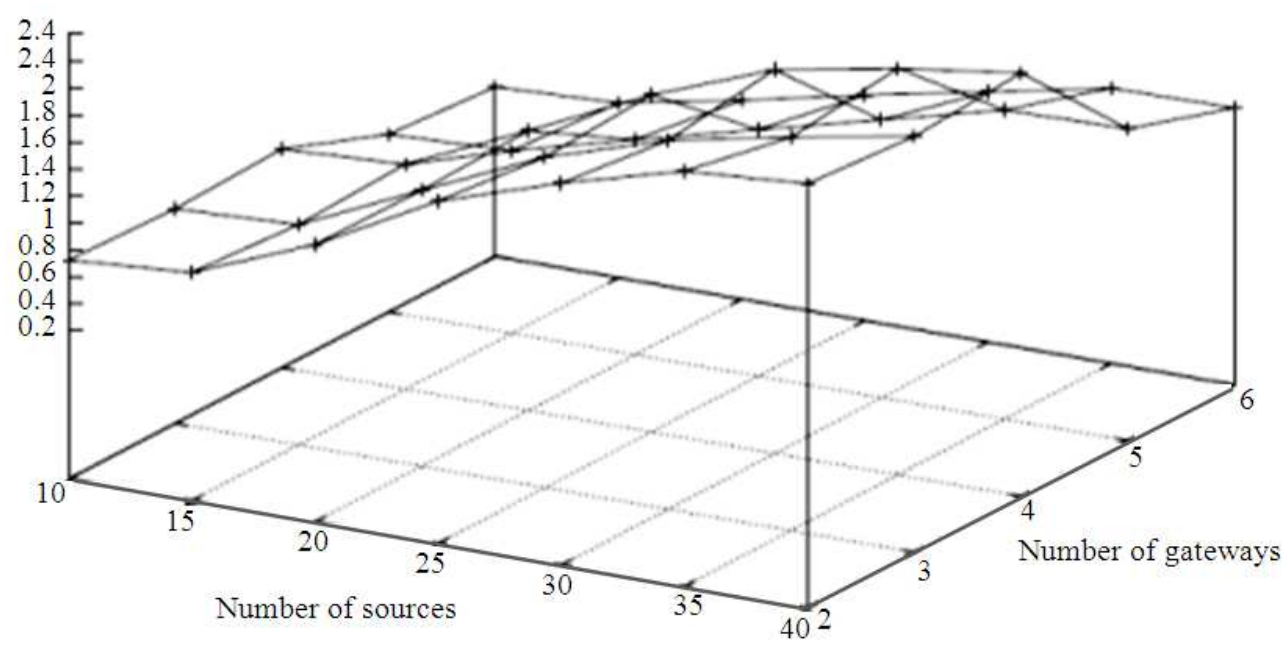

Fig. 2. Mean number of route discoveries per second, $\mathrm{rd}\left(\mathrm{S}, \mathrm{N}_{\mathrm{GW}}\right) .40$ nodes follow the Gauss-Markov mobility pattern at a maximum speed of $20 \mathrm{~m} \mathrm{sec}^{-1}$

The adaptive solution based on maximal source coverage is similar to the hybrid approach, but in this case the TTL $s$ is set to the distance to the farthest source. Let us see a simple example to describe the process of getting the most likely TTL which is used by the algorithm. Let us concentrate on a corner of the lattice, with $\mathrm{N}_{\mathrm{GW}}=1$, $\mathrm{N}_{\text {adhoc }}=5$ and $\mathrm{S}=2$. Obviously, there are two nodes one hop away from the gateway and three nodes at a distance of two hops. Starting with the first source, it can be placed at a distance of 1 hop with a probability $\mathrm{p}(1)=2$ $/ 5$, or at 2 hops with probability p $(2)=3 / 5$.

Assuming that it was placed 1 hop away from the gateway, now we have $\mathrm{p}(1 \mid 1)=1 / 4$ and $\mathrm{p}(2 \mid 1)=3 / 4$ the probabilities for the second source to be at a distance of 1 or 2 hops, respectively given that the first source is at distance 1 hop.

On the other hand, if the first source was placed at a distance of 2 hops, the probabilities for the second source are $p(1 \mid 2)=2 / 4$ and $p(2 \mid 2)=2 / 4$. Therefore, with our maximal source adaptive algorithm in which the selected TTL is set to the number of hops of the furthest away source, the probability to set the TTL of the advertisements to 1 is given by:

$$
P(1) \cdot p(1 \mid 1)=0.1
$$

The probability of setting it to 2 is $p(1)$ :

$$
P(2 \mid 1)+p(2) \cdot p(1 \mid 2)+p(1) \cdot p(2 \mid 2)=0.9
$$

Therefore, the mean TTL is given by $1 \cdot 0.1+2 \cdot 0.9=1.9$.

Generalizing the expression, for each gateway the probability of selecting a particular TTL is given in Equation 7 , being $p(k \mid i, j, \ldots . n-1 \ldots)$ the conditional probability of having the nth source at a distance of $\mathrm{k}$ hops, given that the 1 st source is at $\mathrm{i}$ hops, the 2 nd at $\mathrm{j}$ hops.

In this model, $\mathrm{p}(\mathrm{k} \mid \mathrm{i}, \mathrm{j}, \mathrm{n} .-1 \ldots)$ can be computed as

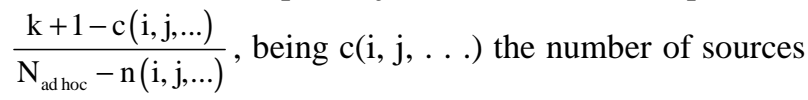
which have been already placed at a distance of $\mathrm{k}$ hops; $\mathrm{n}(\mathrm{i}, \mathrm{j}, \ldots)$ the total number of sources which have been already placed; and $k+1$ is the total number of nodes at a distance of $\mathrm{k}$ hops from the gateway.

I.e., the numerator represents the number of nodes at a distance of $\mathrm{k}$ hops which have not been selected as sources yet and the denominator is the total number of nodes which have not been selected as sources yet. The expression in Equation 7 is just a generalization of the process followed in the previous example:

$$
\begin{aligned}
& P(T T L=s) \\
& =\sum_{i=1}^{s} \sum_{j=1}^{s} S \sum_{k=1}^{s} p(j \backslash i) \operatorname{Sp}(k \backslash i, j, \ldots .), \\
& I=s \backslash j=s \backslash S \backslash k=s
\end{aligned}
$$

The average TTL which is used in proposed adaptive scheme is given by Equation 8. Applying this result to the expression in Equation 6, we get the equation of the overhead caused by the adaptive protocol (Equation 9): 
$\mathrm{S}_{\mathrm{avg}}=\sum_{\mathrm{i}=1}^{\sqrt{\mathrm{N}}-1} \mathrm{i} \cdot \mathrm{P}(\mathrm{TTL}=\mathrm{i})$

$$
\begin{aligned}
& \Omega_{\mathrm{a}}=\Omega_{\mathrm{h}}^{\mathrm{s} a v g}=\lambda_{\mathrm{adv}} \cdot \mathrm{t} \cdot\left(\mathrm{N}_{\mathrm{r}}^{\mathrm{GW}}\left(\mathrm{s}_{\mathrm{avg}}\right)+1\right) \cdot \mathrm{N}_{\mathrm{GW}}+ \\
& \Omega_{\mathrm{r}-\mathrm{gw}} \cdot \mathrm{t} \cdot\left(\mathrm{rd}\left(\mathrm{S}, \mathrm{N}_{\mathrm{GW}}\right)\right) \cdot\left(1-\mathrm{P}_{\mathrm{c}\left(\mathrm{s}_{\mathrm{avg}}\right)}\right)
\end{aligned}
$$

The needed overhead to discover routes to the gateways must be changed. MANET routing is inherently a network layer problem. Typical MANET routing protocols focus on minimizing the number of hops when routing packets to a particular destination. Therefore, when a MANET is connected to the Internet, packets traveling to the Internet are routed to the gateway via the shortest path to minimize routing delay. However, when multiple shortest paths are available to a gateway, the choice of shortest path may become detrimental if a congested path is chosen. Congestion cannot be determined from network layer and it seriously affects the PDR.

\subsection{Metric Based Cross Layer Approach}

In this study use a metric based Link-layer approach to determine congestion that occurs along any path to a gateway.

Contention Metric-One of the major factors creating congestion in a node is contention in the wireless link. In a contention prone channel, Packets cannot readily be forwarded. As a result, packet forwarding is delayed or packets are dropped, minimizing the PDR. To determine this contention formulated in algorithm based on the theory proposed by Karbaschi and Fladenmuller (2005). Here, at each node is measure the contention using Request To Send (RTS) frames, Clear To Send (CTS) frames, data frames and acknowledgment for data frames at the link layer. In a contention free channel, for forwarding a data frame, a single RTS frame is sent followed by the receiving of a single CTS frame followed by the transmission of the data frame and finally receiving an acknowledgement for that data frame. In a contention prone channel any of these four frames can be garbled due to collisions and results in either the retransmission of a RTS frame or retransmission of the data frame or both. Therefore, whenever observe retransmission of a RTS or data frame; finally conclude that contention is occurring. To understand how to calculate the contention, let's assume that an arbitrary node forwards $\mathrm{N}$ frame in $\mathrm{t}$ seconds to its one-hop neighboring nodes. Let $i$ denote the $i_{\text {th }}$ frame being sent from a node to its neighbors. Let the total number of Retransmitted RTS $\left(\mathrm{R}_{\mathrm{RTS}}\right)$ from a node sending $\mathrm{N}$ frames to any arbitrary node within an interval of time $\mathrm{t}$ seconds is named $\mathrm{T}_{\mathrm{RTS}}$, defined as Equation 10:

$$
\mathrm{T}_{\mathrm{RTS}}=\sum_{\mathrm{i}=1}^{\mathrm{N}} \mathrm{R}_{\mathrm{RTS}}(\mathrm{i})
$$

Similarly, let the total number of Retransmitted Data Frames $\left(\mathrm{R}_{\mathrm{DF}}\right)$ from a node sending $\mathrm{N}$ frames to any arbitrary node within an interval of time $t$ seconds is named $\mathrm{T}_{\mathrm{DF}}$, defined as Equation 11:

$$
\mathrm{T}_{\mathrm{DF}}=\sum_{\mathrm{i}=1}^{\mathrm{N}} \mathrm{R}_{\mathrm{DF}}(\mathrm{i})
$$

The interval of time $t$ has been introduced because a node updates its contention metric periodically to reflect the change in the level of contention. The ratio of the total number of failed frames to the total number of frames sent, within an interval of time $t$ seconds, thus reveal a measure of contention in the channel. Let assume that contention ratio $\mathrm{R}_{\mathrm{CON}}$ and define it as Equation 12:

$$
\mathrm{R}_{\mathrm{CON}}=\frac{\mathrm{T}_{\mathrm{RTS}}+\mathrm{T}_{\mathrm{DF}}}{2 \mathrm{~N}+\mathrm{T}_{\mathrm{RTS}}+\mathrm{T}_{\mathrm{DF}}}
$$

Finally, calculate the level of contention at a node and call it Contention Metric (CM), which is in normalized form. A value of $\mathrm{CM}$ of one represents absence of contention around that node. Lower values of CM indicate presence of contention. CM is defined as Equation 13:

$$
\mathrm{CM}=1-\mathrm{R}_{\mathrm{CON}}
$$

Queue metric: Decrease in PDR due to contention is mostly perceived as directly related to reasonably dense networks where nodes are continuously competing to use the channel whenever the channel appears to be free. This is not always true and decrease in PDR between MANETs and Internet, as this study focuses mainly on this flow of PDR, can occur even in sparse networks if some intermediate node along the path to the gateway send packets at a very high rate, thus exhausting the interface packet queue and causing packets to drop. Since used Constant Bit Rate (CBR) for simulation, dropped packets are never retransmitted and critically affect PDR. Higher number of packets in an interface queue of a node increases the chances of exhausting the queue. Therefore the number of packets in a queue at any given node is an important measure in determining congestion along a path, leading to drop in PDR. QM represents the ratio of the queue occupied by packets and can thus can be used to determine congestion as described above. Higher the length of the queue, higher 
the chance of the packets getting dropped, to calculate $\mathrm{QM}$ by taking the average queue length for a period of $\mathrm{t}$ seconds and dividing it by the queue size (i.e., the maximum number of packets that the interface queue can hold at any given instance). Let $\mathrm{L}(\mathrm{t})$ denote the queue length at time $\mathrm{t}$. Let $\mathrm{N}$ be the total number of queue length readings taken within an interval of time $t$ seconds. The average of these queue length readings is given by Equation 14 and 15:

$$
\mathrm{L}_{(\mathrm{t})}=\frac{\sum_{\mathrm{t}=1}^{\mathrm{N}} \mathrm{L}_{(\mathrm{t})}}{\mathrm{N}}
$$

$\mathrm{QM}=\frac{\mathrm{L}_{(\mathrm{t})}}{\mathrm{Q}_{\text {size }}}$

Let $\mathrm{Q}_{\text {size }}$ be the size, in number of packets.

Self Metric- Every node in the MANET measures its own CM and QM at given intervals to determine the level of contention and queue Impediment it is experiencing. Since both $\mathrm{CM}$ and $\mathrm{QM}$ are normalized, they can be combined into a single unit with appropriate weights assigned, Self Metric (SM) define as Equation 16:

$$
\mathrm{SM}=\alpha \times \mathrm{CM}+(1-\alpha) \times \mathrm{QM}
$$

where, $\alpha=0.80$ and $0<\alpha<1$.

Here, alpha is assigned a higher value of 0.80 because it is likely that the number of nodes experiencing contention will be higher compared to the number of nodes experiencing queue impediment due to high packet rates. SM of 1 at any given node means least or no congestion around that node and lower values of SM represents congestion.

Final metric: A node's SM only reveals the contention and queue impediment around and in that node, respectively. But in this study objective is to increase the PDR along a path to the gateway. Therefore, we need a measure of the congestion level of the entire path from a node to the gateway. This is done by introducing another metric, which is called Final Metric (FM). Every node in the MANET calculates its FM by multiplying its own SM with the FM of the node that is the next hop towards the gateway. Figure 3 shows an example with four mobile nodes and how each node calculates it's FM. The FM and SM of the gateway are always set to 1 because it is common for all nodes. At any given time, a node's FM is the product of all the SMs from that node to the gateway. To understand how to measure the FM of a given node, let's assume that a node is the $\mathrm{K}_{\text {th }}$ node from the root (gateway) of the tree, where the root is the first node denoted by $j=0$. Here FM of the Kth node is defined as Equation 17:

$$
\mathrm{FM}_{\mathrm{K}}=\prod_{\mathrm{j}=0}^{\mathrm{K}} \mathrm{SM}_{\mathrm{j}}
$$

FM of any node reveals the level of congestion, of the entire path, from that node up to the gateway. Thus, it can be used in determining the least congested path when multiple paths are available to reach the gateway. This information can help to optimize a network layer. GC REQ messages are only sent by nodes outside a proactive zone. They are flooded to the whole reactive zone and therefore there is as much forwarding as nodes in that zone. So, the overhead is given by the number of nodes which are placed outside the proactive zone, $\mathrm{N}_{\text {pz_out }}=\mathrm{N}_{\text {adhoc }}-\mathrm{N}_{\mathrm{GW}} \cdot \mathrm{N}_{\mathrm{r}}^{\mathrm{GW}}$ ( $\mathrm{s}_{\mathrm{avg}}$ ). The GC REP is sent by the nodes placed just in the border of a proactive zone. The number of such nodes can be computed as $\mathrm{N}_{\text {pz_broder }}=$ $\mathrm{N}_{\mathrm{GW}} \cdot \mathrm{N}_{\mathrm{r}}^{\mathrm{GW}}\left(\mathrm{s}_{\mathrm{avg}}\right)-\mathrm{N}_{\mathrm{r}}^{\mathrm{GW}}\left(\mathrm{s}_{\mathrm{avg}}-1\right)=\mathrm{N}_{\mathrm{GW}} \cdot\left(\mathrm{s}_{\mathrm{avg}}+1\right)$. Combining expressions, the expected overhead per each source which does not receive periodic GC REP messages is given by Equation 18 and the total overhead of proposed Contention Aware QoS Adaptive Protocol scheme is in Equation 19:

$$
\begin{aligned}
& \Omega_{\mathrm{p}_{-} \mathrm{gw}}=\mathrm{N}_{\mathrm{pz}_{-} \text {out }}+\mathrm{N}_{\mathrm{pz}_{-} \text {border }}=\mathrm{N}_{\mathrm{ad} \mathrm{hoc}} \\
& +\mathrm{N}_{\mathrm{GW}} \cdot\left[\left(\mathrm{s}_{\mathrm{avg}}\right)+1-\mathrm{N}_{\mathrm{r}}^{\mathrm{GW}}\left(\mathrm{s}_{\mathrm{avg}}\right)\right] \\
& \Omega_{\mathrm{CAQAP}}=\lambda_{\text {avg }} \cdot \mathrm{t} \cdot\left(\mathrm{N}_{\mathrm{r}}^{\mathrm{GW}}\left(\mathrm{s}_{\mathrm{avg}}\right)+1\right) \cdot \mathrm{N}_{\mathrm{GW}} \\
& +\Omega_{\mathrm{p}-\mathrm{gw}} \cdot \operatorname{tr}\left(\operatorname{rd}\left(\mathrm{S} \cdot \mathrm{N}_{\mathrm{GW}}\right)\right) \cdot\left(1-\mathrm{P}_{\mathrm{c}\left(\mathrm{s}_{\text {avg }}\right)}\right)+\prod_{\mathrm{j}=0}^{\mathrm{K}} \mathrm{SM}_{\mathrm{j}}
\end{aligned}
$$

Finally, obtain an expression for the overhead caused by the Quality of Service solution, the model predicts a good Contention Aware QoS based Adaptive Protocol discovery scheme both with respect to Intra-MANET and Internet-MANET.

\section{SIMULATION AND RESULT ANALYSIS}

In this section used for NS2 simulation and implemented the hybrid, adaptive and Contention Aware QoS Adaptive Protocol (CAQAP) and appropriate modifications to the MAC sub layer in NS2 to achieve cross-layer optimization in CAQAP by sending the count of data frames, retransmitted data frames, RTS frames and retransmitted RTS frames from the link layer to the network layer. 


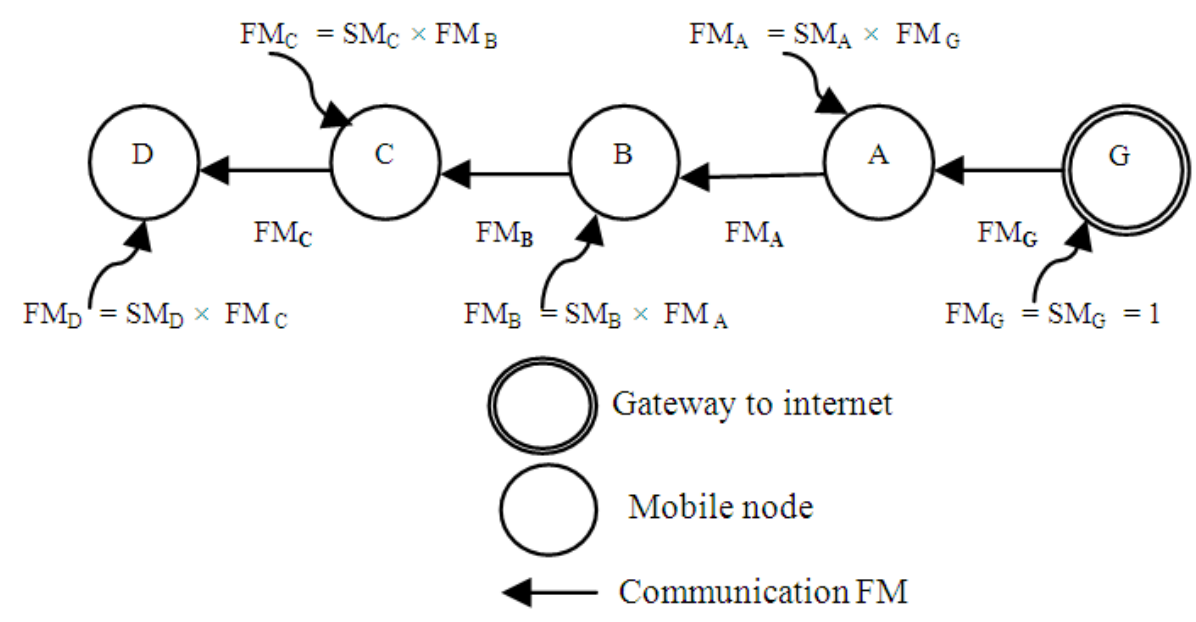

Fig. 3. Shows an example with four mobile nodes and how each node calculates it's FM

Compared the control packet overhead and PDR for the three protocols, hybrid, adaptive and CAQAP and showed that CAQAP protocol performs better in terms of PDR in various scenarios. In this simulated network had 10 to 50 nodes with run time of 600 simulated seconds. Parameter values used for Random way point mobility model, MAC802.11 link layer protocol, 1Mbps wireless bandwidth, transmission wireless range of $250 \mathrm{~m}$, Interface queue length is 50 packets, Interface queue type is used droptail and grid size is $1400 \times 1400 \mathrm{~m}$. Averages of ten simulations were taken for each data point. On the average, Internet-MANET packets constituted $44 \%$ of the traffic and Intra-MANET packets constituted 54\% of the traffic. Packet size was set at 512 bytes and nodes sent CBR packets with rates ranging from 10 packets/s to 35 packets/s from each source. Node speeds were varied from 0 to $10 \mathrm{~m} \mathrm{sec}^{-1}$. Even though all simulations had both Internet-MANET traffic and Intra-MANET traffic flowing simultaneously, the data obtained for each type of traffic were shown separately to demonstrate their individual performance.

Effective in the number of nodes: Internet-MANET traffic, CAQAP packets were always routed via the least congested path when traveling to and from the gateway and as a result, even with increase in congestion and contention due to increase in number of nodes, the PDR doesn't considerably drop. Adaptive, however, suffers from the increase in number of nodes because of increased contention and congestion and being unable to determine it. PDR in hybrid, even in a small MANET, is not very high as considerable amount of the bandwidth is occupied with control packets; especially around the gateway. In Fig. 4 and 5, show the all three protocols, packets traveling within the MANET are routed without consideration of congestion or Contention, therefore the protocols route packets using the shortest path. The slight drop in PDR for all three protocols occurs due to contention arising from higher number of nodes.

Effective in the speed of nodes: In Fig. 6 and $\mathbf{7}$ show that at low mobility CAQAP performs better than hybrid because received. In Fig. 6, performance of both CAQAP and adaptive falls at higher speeds as breakage in tree paths towards gateway usually requires longer time to repair compared to paths broken in hybrid. In Fig. 7, at higher Speeds, the PDR drops significantly in all three protocols because routes are continuously broken and repaired at multiple points in the MANET. Control overhead and faster tree repair. Faster tree repair was achieved by; unlike adaptive, reducing the time between heartbeat intervals when a heartbeat acknowledgement is not consumption by control packets is less than hybrid. CAQAP also performs slightly better than Adaptive because of lower at low mobility tree formation is stable and bandwidth.

Effective in the pause time: In Fig. 8 and 9, show that at lower pause time, hybrid performs better than both adaptive and CAQAP. This is because, both adaptive and CAQAP are tree based network and a connection lost between a parent and child near the root of the tree results in rebuilding of all the connections in that branch under that node. In higher pause times, as mobility is low, such breakages in branches do not occur frequently and higher PDR is obtained. 
Palani, K. and P. Ramamoorthy / Journal of Computer Science 9 (10): 1329-1340, 2013

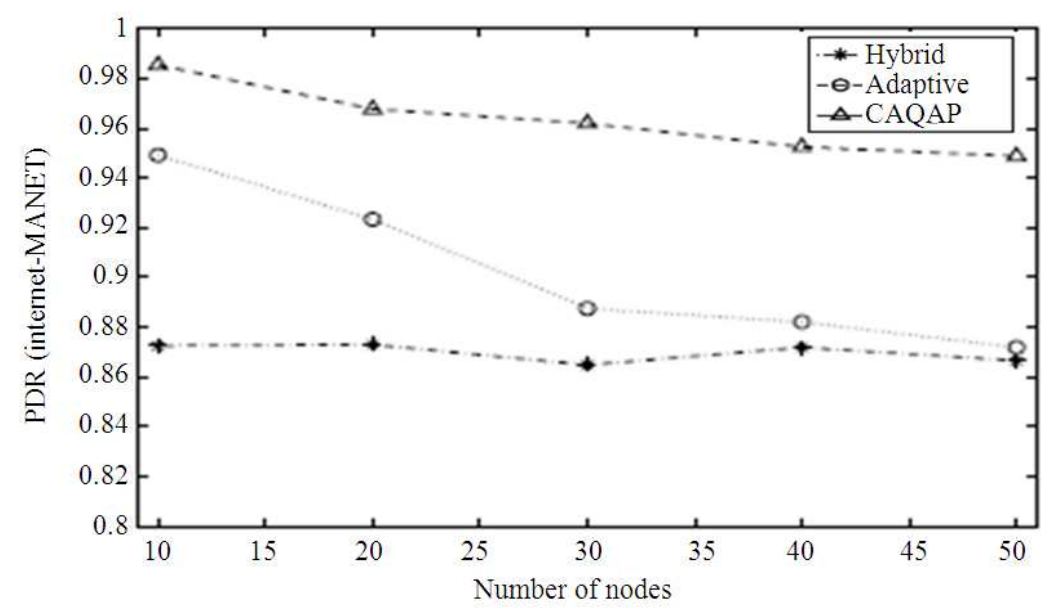

Fig. 4. Effect on internet-MANET connectivity with number of nodes

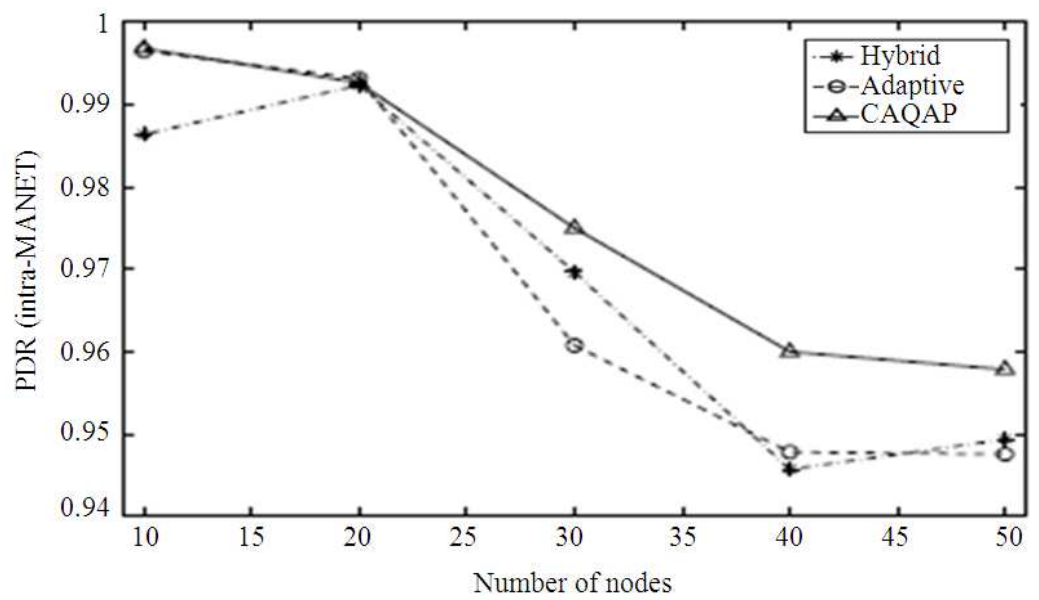

Fig. 5. Effect on intra-MANET connectivity with number of nodes

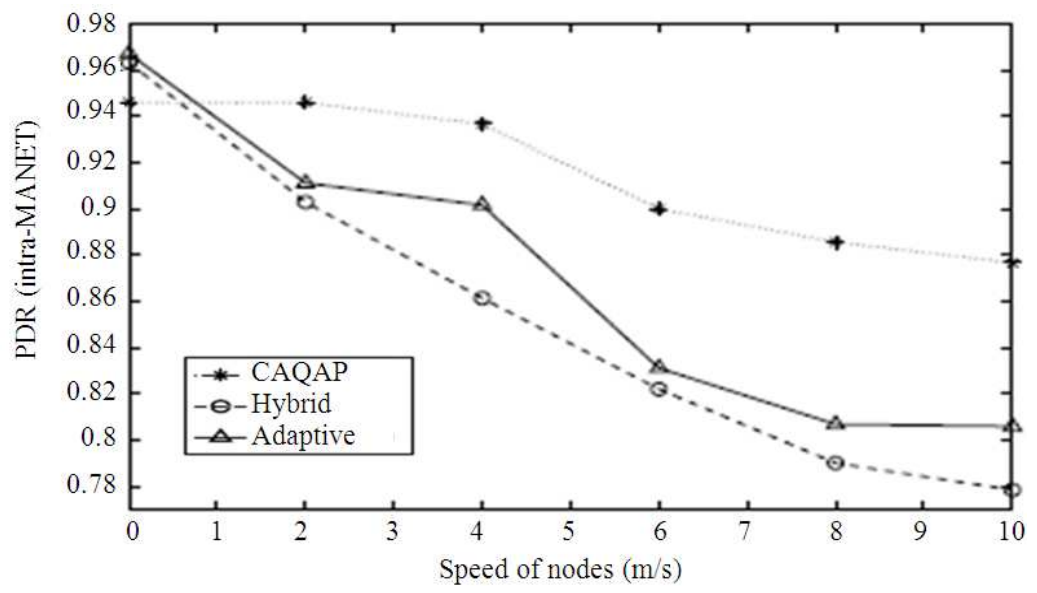

Fig. 6. Effect on internet-MANET connectivity with speed of nodes $(\mathrm{m} / \mathrm{s})$ 
Palani, K. and P. Ramamoorthy / Journal of Computer Science 9 (10): 1329-1340, 2013

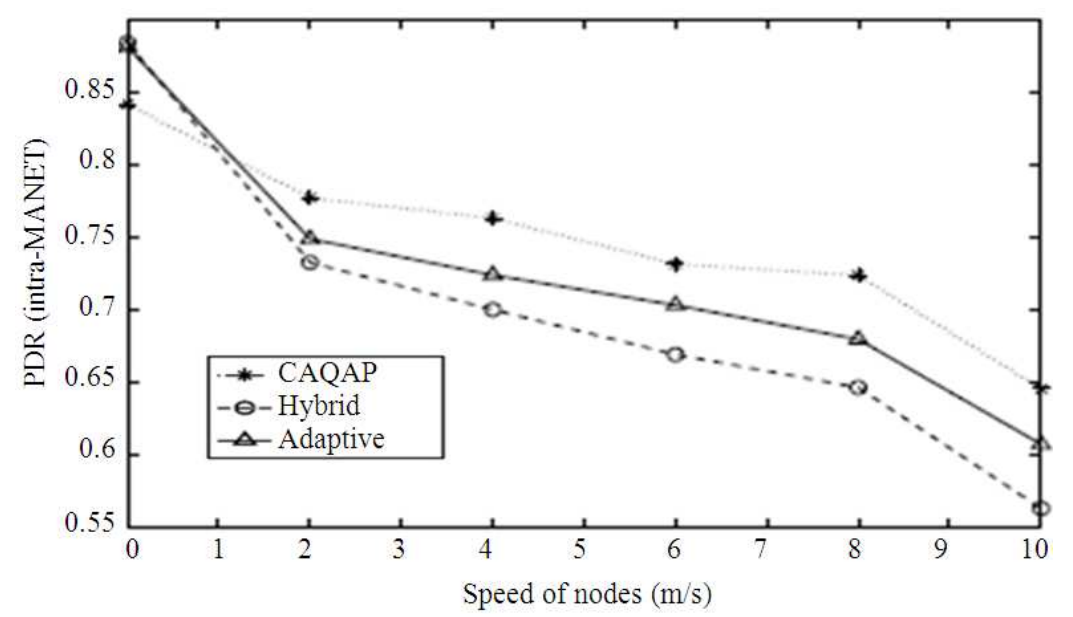

Fig. 7. Effect on intra-MANET connectivity with speed of nodes $(\mathrm{m} / \mathrm{s})$

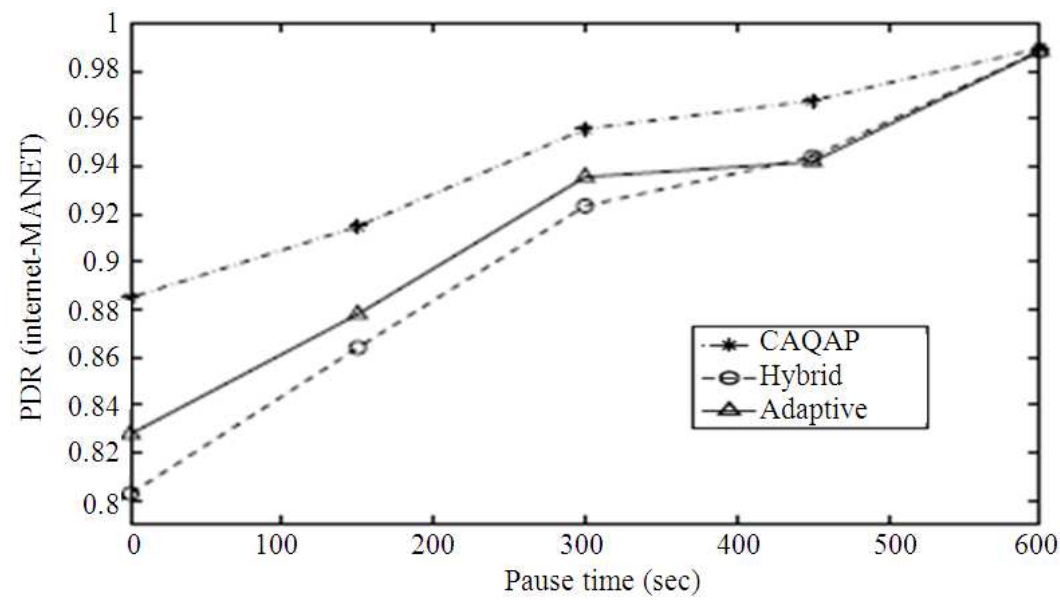

Fig. 8. Effect on internet-MANET connectivity with pause time (sec)

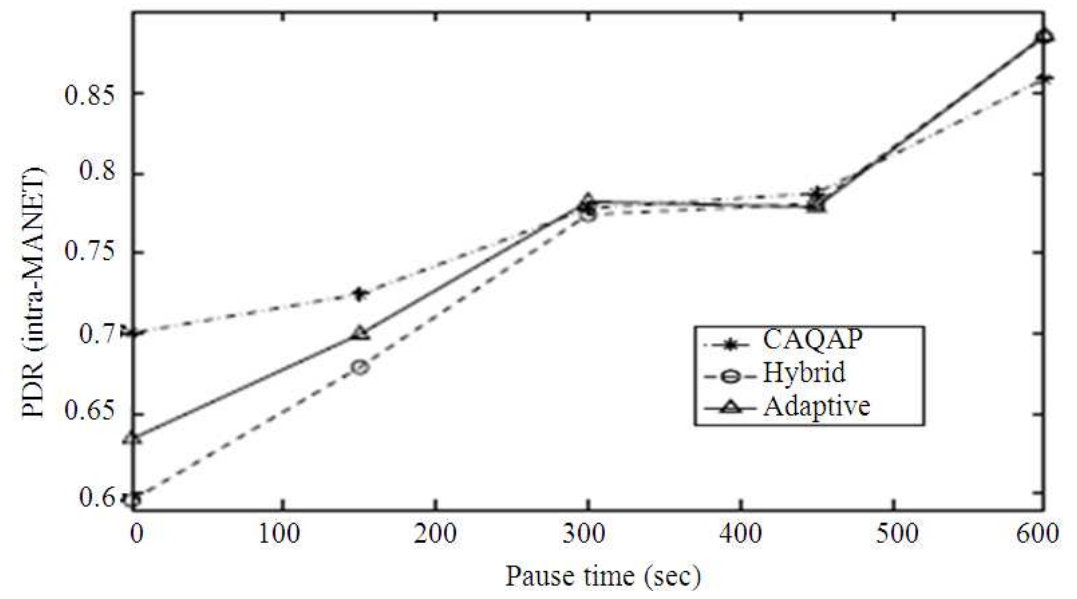

Fig. 9. Effect on intra-MANET connectivity with pause time (sec) 


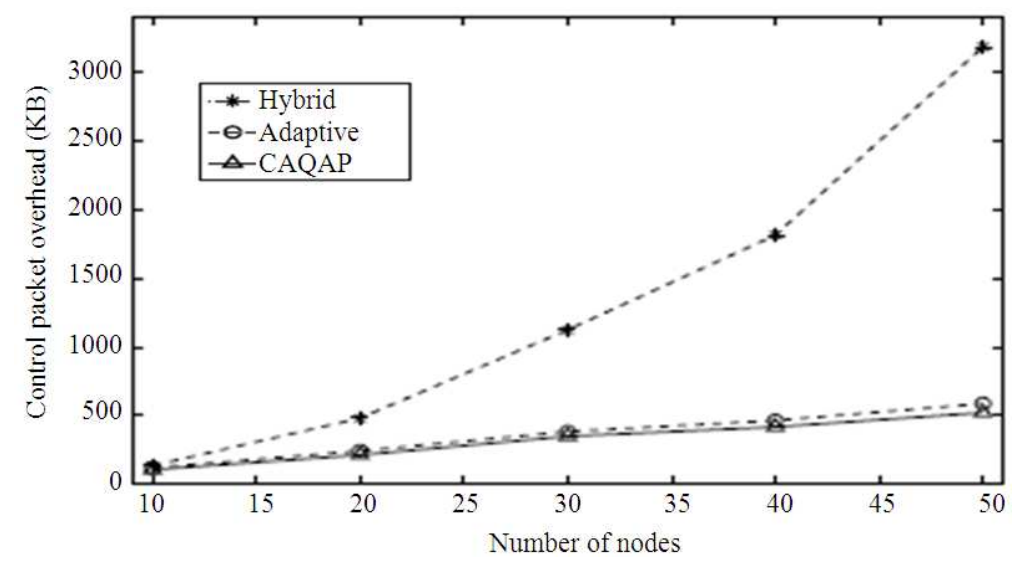

Fig. 10. Effect on control packet overhead

Effective in the control packet overhead: Control packet overhead is defined as the number of control packets transmitted by a MANET protocol. Higher bandwidth occupancy by control packets usually results in low PDR, in this work used the control packet overhead in Kilo Bytes (KB) because it is a more accurate measure when control packet size varies Fig. 10 shows the control packet overhead against the number of nodes for each protocol.

Control packet overhead in hybrid increase rapidly with the increase number of nodes, because in hybrid each node must store routes to all other nodes in the MANET and share this routing information at intervals with its one-hop neighbors. Control packet overhead of CAQAP and adaptive is less because only small sized packed and small size acknowledgement messages are sends to maintain MANET overlay. Control packet overhead of CAQAP is slightly less than adaptive.

\section{CONCLUSION}

In this study shown that Metric based cross-layer approach performs better in Contention Aware QoS Adaptive Protocol based MANET overlay. Where obtaining Internet connectivity over a large area is a challenge and data loss can be crucial in hampering day-to-day operations, CAQAP can provide global connectivity and ensure high packet delivery ratio for both Internet-MANET and Intra-MANET traffic. Results show that CAQPA performs better than both adaptive and hybrid in various situations including low mobility and varied number of nodes, with shown results for PDR of Internet-MANET and Intra-MANET routing separately to better understand how each flow of data is affected in different situations.

\section{REFERENCES}

Akyildiz, I.F., J, Xie and S. Mohanty, 2004. A survey of mobility management in next-generation all-IPbased wireless systems. IEEE Wireless Commun., 11: 16-28. DOI: 10.1109/MWC.2004.1325888

Ammari, H. and H. El-Rewini, 2004. Integration of mobile ad hoc networks and the Internet using mobile gateways. Proceedings of 18th International Parallel and Distributed Processing Symposium, Apr. 26-30, IEEE Xplore Press, pp: 3007-3014. DOI: 10.1109/IPDPS.2004.1303253

Bin, S., S. Bingxin, L. Bo, H. Zhonggong and Z. Li, 2005. Adaptive gateway discovery scheme for connecting mobile ad hoc networks to the internet. Proceedings of the International Conference on Wireless Communications, Networking and Mobile Computing, Sep. 23-26, IEEE Xplore Press, pp: 795-799. DOI: 10.1109/WCNM.2005.1544172

Denko, M.K., E. Shakshuki and H. Malik, 2007. A mobility-aware and cross-layer based middleware for mobile ad hoc networks. Proceedings of the $21 \mathrm{st}$ International Conference on Advanced Information Networking and Applications, May 21-23, IEEE Xplore Press, Niagara Falls, ON, pp: 474-481. DOI: 10.1109/AINA.2007.15

Ding, S., 2008. A survey on integrating MANETs with the internet: Challenges and designs. Comput. Commun., 31: 3537-3551. DOI: 10.1016/j.comcom.2008.04.014

Ergen, M. and A. Puri, 2002. MEWLANA-mobile IP enriched wireless local area network architecture. Proceedings of the 56th Vehicular Technology Conference, Sep. 24-28, IEEE Xplore Press, pp: 2449-2253. DOI: 10.1109/VETECF.2002.1040661 
Hamadan, A., U. Korner and A. Nilsson, 2003. A study of internet connectivity for mobile ad hoc networks in NS2. Department of Communication Systems, Lund Institute of Technology, Lund University.

Hoang, V.D., Z. Shao, M. Fujise and H.M. Nguyen, 2004. A novel solution for global connectivity in MANET. Proceedings of the IEEE 60th Vehicular Technology Conference, Sep. 26-29, IEEE Xplore Press, pp: 2819-2823. DOI: 10.1109/VETECF.2004.1400573

Hwang, R.H., C.Y. Li, C.Y. Wang and Y.S. Chen, 2005. Mobile IPv6-based ad hoc networks: Its development and application. IEEE J. Selected Areas Commun., 23: 2161-2171. DOI: 10.1109/JSAC.2005.856826

Jonsson, U., F. Alriksson, T. Larsson, P. Johansson and G.Q. Maguire, 2000. MIPMANET-mobile IP for mobile ad hoc networks. Proceedings of the 1st Annual Workshop on Mobile Ad Hoc Networking Computing, Aug. 11-11, IEEE Xplore Press, Boston MA., $\quad$ pp: 75-85. DOI: 10.1109/MOBHOC. 2000.869215

Karbaschi, G. and A. Fladenmuller, 2005. A link-quality and congestion-aware cross layer metric for multihop wireless routing. Proceedings of the IEEE International Conference on Mobile Ad Hoc and Sensor Systems, Nov. 7-7, IEEE Xplore Press, Washington, DC., pp: 649-655. DOI: 10.1109/MAHSS.2005.1542855

Kiess, W. and M. Mauve, 2007. A survey on real-world Implementations of mobile ad-hoc networks. Ad Hoc Netw., 5: 324-339. DOI: 10.1016/j.adhoc.2005.12.003

Lee, J., D. Kim, J.J. Garcia-Luna-Aceves, Y. Choi and J. Choi et al., 2003. Hybrid gateway advertisement scheme for connecting mobile ad hoc networks to the internet. Proceedings of the 57th Vehicular Technology Conference, Apr. 22-25, IEEE Xplore Press, $\quad$ pp: 191-195. DOI: 10.1109/VETECS.2003.1207528

Nilsson, A., C.E. Perkins, A.J. Tuominen, R. Wakikawa and J.T. Malinen, 2002. AODV and IPv6 internet access for ad hoc networks. ACM SIGMOBILE Mobile Comput. Commun. Rev., 6: 102-103. DOI: $10.1145 / 581291.581310$
Perkins, C.E., 1996. Mobile-IP, ad-hoc networking and nomadicity. Proceedings of 20th International Computer Software and Applications Conference, Aug. 21-23, IEEE Xplore Press, Seoul, pp: 472-476. DOI: 10.1109/CMPSAC.1996.544615

Ratanchandani, P. and R. Kravets, 2003. A hybrid approach to internet connectivity for mobile ad hoc networks. Proceedings of the IEEE Wireless Communications and Networking, Mar. 20-20, IEEE Xplore Press, New Orleans, LA., USA., pp: 1522-1527. DOI: 10.1109/WCNC.2003.1200612

Ruiz, P. and A. Gomez-Skarmeta, 2005. Adaptive gateway discovery mechanisms to enhance internet connectivity for mobile ad hoc networks. Ad Hoc Sensor Wireless Netw., 1: 159-177.

Song, W. and X. Fang, 2006. Routing with congestion control and load balancing in wireless mesh networks. Proceedings of the 6th International Conference on ITS Telecommunications, Jun. 2123, IEEE Xplore Press, Chengdu, pp: 719-724. DOI: 10.1109/ITST.2006.289002

Sun, Y., E.M. Belding-Royer and C.E. Perkins, 2002. Internet connectivity for ad hoc mobile networks. Int. J. Wireless Inform. Netw., 9: 75-88. DOI: 10.1023/A:1015399632291

Tseng, Y.C., C.C. Shen and W.T. Chen, 2003. Integrating mobile IP with ad hoc networks. Computer, 36: 48-55. DOI: 10.1109/MC.2003.1198236

Wakikawa, R., J.T. Malinen, C.E. Perkins, A. Nilsson and A.J. Tuominen, 2006. Global connectivity for IPv6 mobile ad hoc networks. Mobile Ad Hoc Network.

$\mathrm{Xi}$, J. and C. Bettstetter, 2002. Wireless multihop internet Access: Gateway discovery, routing and addressing. Proceedings of the International Conference on Third Generation Wireless and Beyond, May 28-31, San Francisco, CA, USA., pp: 109-114. 